\title{
Turn-signal utilization by rats with either unilateral or bilateral posterior parietal cortex injuries
}

\author{
WILLIAM F. MCDANIEL, LAURA B. WILLIAMS, CULLEN M. ATTAWAY, and DAVID M. COMPTON \\ Georgia College \& State University, Milledgeville, Georgia
}

\begin{abstract}
Rats with sham, unilateral, or bilateral aspiration lesions of the posterior parietal cortex (PPC) were trained in a water T-maze to use flashing lights located along the starting alley (two each) and inner walls of the goal alleys (two each) to find a hidden escape platform. Thereafter, the performance of the spatial response was tested under several conditions. Rats with bilateral PPC lesions were significantly inferior to unilateral- and sham-injured rats in learning the "turn-signal" cued spatial task. Also, leftPPC-injured rats committed significantly more errors than did the control animals. After mastering the task, cue saliency was reduced and the amount of spatial discontiguity between the stimuli and escape site was increased in two stages. That is, the flashing light closest to the escape platform was turned off for one testing session. On the following day, animals were required to rely on information located within the starting alley to make the correct spatial response at the choice point. Animals with bilateral or left PPC lesions were significantly impaired on the task with cues located only in the starting alley. The animals were then tested with competing constant illumination of the lights on the side of the apparatus opposite the flashing-light cues. The performance of all animals dropped to chance and failed to improve with training. Finally, three of the sham-operated controls were retrained to criterion on the original discrimination and prepared with bilateral PPC injuries. Substantial savings was observed. The results reveal a greater role of the left PPC than the right in the use of local sensory cues for spatial navigation, and they show that the PPC is not the repository of the "engram" for this learned visuospatial behavior.
\end{abstract}

Research conducted during the past 20 years has shown that the rat has a cortical territory similar in many respects to posterior parietal cortex (PPC) in humans and nonhuman primates (e.g., Burcham, Corwin, Stoll, \& Reep, 1997; Kolb, 1990; Kolb, Buhrmann, McDonald, \& Sutherland, 1994; Kolb \& Walkey, 1987; McDaniel, 1985; McDaniel \& Wall, 1988). Anatomical homology is supported by similarities in the patterns of connections in rat and monkey. Behavioral analogy is supported by the observation that lesions within these territories result in similar kinds of behavioral dysfunctions. Since the PPC in rat receives cortico-cortical afferents from many areas, including the temporal, somatosensory, visual, and frontal regions (Kolb \& Walkey, 1987), it appears to constitute a multimodal region that may be involved in forming associations between stimuli and in guiding the motor systems. Neurophysiological studies of a region that appears to overlap with the PPC have shown that many neurons are responsive to visual stimuli, and they demonstrate movement selectivity characteristics for specific head directions (Chen, Lin, Barnes, \& McNaughton, 1994; Chen, Lin, Green, Barnes, \& McNaughton, 1994; McNaughton, Leonard, \& Chen, 1989). In the monkey, many neurons within PPC discharge only when a sensory stimulus of sufficient interest is placed in a specific spatial lo-

Correspondence should be addressed to W. F. McDaniel, Department of Psychology, Georgia College \& State University, Milledgeville, GA 31061-0490 (e-mail: bmcdanie@mail.gac.peachnet.edu). cation consistent with the monkey's gaze and reach ( $\mathrm{Hy}$ värinen \& Poranen, 1974). Thus, it might be expected that homologous neurons within the PPC would play an important role in spatial navigation by quadrupedal animals, such as the rat, where the sensory apparati of the nose and mouth, rather than the hand or paw, play an important role in the investigation of the environment. The neurophysiological observations in the rat are also consistent with impressions derived from studies using electrophysiological and blood-flow procedures with both humans and nonhuman primates (reviewed by Kolb \& Whishaw, 1996, pp. 268-271). That is, they support the suggestion that the PPC in the rat plays a role in visuomotor guidance, as is the case in humans and nonhuman primates.

Prior to the publication of what we consider to be a "landmark" study by Kolb and Walkey (1987), the territory comprising the PPC in the rat was only vaguely defined. In many of the early publications from this laboratory (see McDaniel, 1985, for a review), the territory was labeled parietal association cortex (PAC). Although our lesions often included what is now defined as PPC, like those of Kolb, Sutherland, and Whishaw (1983), who reported that rats with parietal lesions were relatively unimpaired in Morris water maze and radial arm maze tasks, they also often spared much of the PPC while invading the hindlimb region and PAR 1 (Zilles, 1985). Our choice of the term PAC was based on the limited neuroanatomical evidence (e.g., Lashley, 1941; McDaniel, McDaniel, \& Thomas, 1978) and limited neurobehavioral evidence 
concerning the effects of small lesions in this tegion upon behavior (Boyd \& Thomas, 1977; McDaniel \& Thomas, 1978; Thomas \& Weir, 1975) available at the time. These studies, along with studies in other laboratories (e.g., Thompson, Huestis, Crinella, \& Yu, 1986; Thompson \& Yu, 1985), showed that bilateral PAC lesions resulted in disturbed spatial learning in a variety of mazes (e.g., Hebb-Williams, Lashley III mazes) or mazelike (e.g., Y- or T-maze spatial position reversal and spatial alternation; Kolb et al., 1983; McDaniel, Davall, \& Waters, 1989; McDaniel \& Thomas, 1978) tasks. Recently, the influences of similar lesions have been extended to the Maier 3-table task (Thinus-Blanc, Save, Poucet, \& Foreman, 1996). In this study, and in all others, with extended training the animals with PAC injuries eventually mastered the tasks. This result supports the view expressed by several researchers that, although some neural territories may play a more important role in particular aspects of spatial function than others (Burcham et al., 1997; Kolb, et al., 1994), significant compensation for visuospatial disturbances can occur with the passage of time or with involvement in training (Kolb et al., 1994; Kolb \& Walkey, 1987; McDaniel et al., 1995; McDaniel \& Wall, 1988).

On the basis of their anatomical investigations of cortico-cortical and thalamic connections, Kolb and Walkey (1987) more precisely defined the territory corresponding to $P P C$ in the rat as lying $4.0-6.0 \mathrm{~mm}$ posterior to bregma and extending laterally with respect to midline 2.0 to approximately $6.0 \mathrm{~mm}$. Later, Chandler, King, Corwin, and Reep (1992) and Reep, Chandler, King, and Corwin (1994) reevaluated the thalamic connections of PPC and provided a refinement of the coordinates. They suggested that the PPC in the rat corresponds to a thin strip of tissue located 3.4-4.4 mm posterior to bregma and extending laterally from midline $1.5-4.5 \mathrm{~mm}$. This territory corresponds roughly to area 7 , as identified by Vogt and Miller (1983). Obviously one major problem in this area of research relates to the exact location of the cortical territory injured in various studies. Most studies, even with attempts to restrict lesions to the territories defined by Kolb and Walkey and by Burcham et al., have utilized lesions extending somewhat rostrally and caudally beyond these limits.

Recent neurobehavioral studies with more caudally placed lesions and with more refined behavioral methodologies for studying spatial navigation and spatial learning have shown that bilateral PPC lesions seriously impair spatial navigation by disturbing the use of allocentric, or environmental, visual cues (King \& Corwin, 1992; Kolb et al., 1994; Kolb \& Walkey, 1987). As might be expected, given the neurophysiological results described previously, rats with bilateral PPC injuries also make initial heading errors and have curved trajectories in open-field navigation environments, such as the Morris water-maze problem (Kolb \& Walkey, 1987). Yet, considerable spatial ability survives PPC lesions. For example, bilateral PPC lesions do not disturb the acquisition of a multiple-
T water maze where extramaze visual cues apparently play a minimal role (McDaniel, Compton, \& Smith, 1994). After learning this task, the barriers composing the multiple- $T$ water maze were removed from the elliptical tank, and the animals were tested for a "cognitive map" of the environment. PPC-injured rats navigated to the hidden escape platform as effectively as control animals. Rats with hippocampal lesions not only demonstrated a severe learning deficit in this task but, after finally learning the task, they also failed to demonstrate a cognitive map when the multiple-T walls were removed. Thus, we have concluded that the PPC, while an important part of the cognitive mapping system of the brain (DiMattia \& Kesner, 1988a, 1988b), is not the repository of the environmental map "engram." Similar results have been reported in a study using a shock-motivated 14-unit T-maze (Jucker, Kametani, Bresnahan, \& Ingram, 1990). That is, retention of the maze habit was unimpaired in the animals sustaining extensive bilateral parietal injuries, but retention was severely impaired in animals with parietal lesions that included substantial invasion of the hippocampal formation. Also, we have demonstrated that the impaired learning of difficult visuospatial relationships (e.g., if approaching a dark visual stimulus in a $\mathrm{T}$ water maze, turn right, but if approaching a white stimulus, turn left) can be reversed by imposing a response cost for errors (cf. Davis \& McDaniel, 1993, vs. McDaniel \& Skeel, 1993). Results such as these have led some authors (DiMattia \& Kesner, 1988a, 1988b; McDaniel et al., 1995; Save, Buhot, Foreman, \& Thinus-Blanc, 1992; Save, Poucet, Foreman, \& Buhot, 1992) to conclude that the PPC is especially involved in the "effortful processing of spatial relations" (Save, Buhot, et al., 1992, p. 125).

Several studies have reported that different behavioral sequelae accompany right as opposed to left parietal lesions. For example, right parietal lesions result in increased activity in open-field testing (Crowne, Richardson, \& Dawson, 1987). Also, studies conducted in two laboratories (Crowne, Novotny, Maier, \& Vitols, 1992; King \& Corwin, 1992) have shown that rats with right, but not left, PPC injuries show performance deficits in tasks placing significant demands on the use of allocentric visual targets for successful navigation (e.g., cheeseboard maze, and the landmark navigation task in the Morris water maze).

McDaniel et al. (1995) investigated the impact of unilateral and bilateral PPC lesions on spatial navigation in a Greek-cross-shaped water maze. Different groups of animals were trained on either a place habit or a response habit in an environment with many extramaze visuospatial cues. In the place-learning paradigm, the animals had to swim from one of two opposed starting points to a single escape site. In the response-learning paradigm, the animals had to make a particular turn (i.e., left for some animals, right for others) at the choice site. All animals, including those with bilateral PPC lesions, learned the place habit in the Greek-cross environment rapidly, and there were no differences between the lesion groups 
and the sham control rats. Thus, consistent with Save, Buhot, et al. (1992), we concluded that the integrity of the PPC was not necessary for successful navigation on the basis of simple visuospatial relationships. In the responselearning paradigm, both the bilateral-PPC-injured and the right-PPC-injured rats were significantly inferior to the left-PPC-injured rats and the sham-operated controls. We had originally hypothesized that a double dissociation would be observed in these tasks, with right-PPCinjured rats showing deficient performance in the placelearning task and left-PPC-injured rats showing a deficit in the response-learning task. Our results forced us to rethink the demands of the training paradigms. It occurred to us that the response-learning paradigm could be learned by two rules. One rule, certainly the one that seems simplest, would dictate that the animal always make a particular $90^{\circ}$ turn at the choice site. However, the task could also be solved by forming two nonconceptual conditional associations, or perceptual rules. These might be stated as follows: If approaching visual cues available from start box 1, approach visual cues associated with goal box 1 ; however, if approaching visual cues available from start box 2, approach the cues associated with goal box 2 . Thus, we suggested that perhaps injuries in either the right or the left PPC could disturb egocentric abilities. However, in the presence of allocentric visual cues, leftPPC-injured animals might be more capable of compensating for deficient egocentric mechanisms by utilizing allocentric visual to guide successful spatial navigation. We tested this hypothesis in another experiment using the Greek-cross response-learning paradigm but in the absence of reliable visual cues. The apparatus was surrounded by a white cheese-cloth curtain, illumination was provided by red overhead lights, and the spatial positions of the two trainers (the only possible visuospatial cues) were randomized across trials. Under these conditions, the left-PPC-injured rats were as deficient as the right-PPCinjured rats.

Our suggestion that injuries to the PPC in either hemisphere can impair egocentric spatial behavior appears to contradict the results obtained by King and Corwin (1992) and Kolb et al. (1994). Each of these studies showed that PPC-injured rats perform as well as control animals in tasks placing significant demands on egocentric mechanisms. Both investigations used novel radial-arm-maze methodologies. King and Corwin modeled the procedure originally utilized by Kesner, Farnsworth, and DiMattia (1989), where, after finding food in an alley on the initial foraging venture, the animal could find food only in the adjacent arms next to the initially selected arm on the next venture. Kolb et al. started the rat in a goal arm and required that the rat choose the baited adjacent arm on the right. In both paradigms, animals with prefrontal or medial agranular lesions proved to be deficient. However, rats with PPC lesions performed similar to normal controls.

A recent study by Save and Moghaddam (1996) has reopened the question of a role of parietal cortex in ego- centric processes. Their study revealed that rats with bilateral injuries within the associative parietal cortex (APC), a territory that appears to include PPC, suffer severe "egocentric" deficits reflected in the processing of "kinesthetic information." Specifically, these investigators prepared rats with bilateral APC lesions and trained them in total darkness to swim to an escape platform in a Morris water maze. The start point and the escape point were different across training sessions and days; however, the relative positions of the start point and the escape platform were constant throughout training. Although the APC-injured rats performed better across trials, their heading errors and escape latencies were consistently inferior to those of the control animals. These observations led the investigators to conclude that APC plays an important role of an "interface between the egocentric and allocentric coding, first by integrating kinesthetic information and second by incorporating some visual information" (Save \& Moghaddam, 1996, p. 84). This conclusion is consistent with that derived by McDaniel et al. (1995), who proposed that PPC in both hemispheres plays an important role in egocentric processes, but the right PPC also plays a specialized role in comprehending the visuospatial information necessary for the reliable use of complex allocentric cues. Finally, using both unilateral spreading cortical depression and unilateral whisker anesthesia, LaMendola and Bever (1997) have recently shown that rats learn a foraging pattern in the eight-arm radial maze better with an intact left hemisphere and functioning right-sided whiskers than with an intact right hemisphere and functioning left-sided whiskers. The authors conclude that their results are consistent with clinical observations made in humans. That is, the left hemisphere may play a greater role in coding "specific local aspects and features" of the environment, whereas the right hemisphere is more involved in coding the "global and metric aspects of a spatial array" (LaMendola \& Bever, 1997, p. 485).

The purpose of the present experiment was to explore further the different, but complimentary, functions of the PPC in the two hemispheres. To that end, rats were prepared with either sham, bilateral, unilateral left, or unilateral right PPC lesions and were trained to execute either right or left turns in a water T-maze on the basis of the relative positions of "local" flashing "turn-signal" light cues. The flashing lights were located along the inner walls of the start alley (two each) and the inner walls of the goal alleys (two each). The lights in the goal arms were spatially discontinguous with respect to the escape platform at the end of the alley. Flashing lights in the right visual field signaled that the platform was located in the right arm of the maze, and flashing lights in the left visual field signaled that the platform was located in the left arm. Once learned, performance was tested under conditions of reduced cue saliency and in the presence of competing stimuli. Performance of this habit, based mainly or solely on information in the starting alley, would place heavy demands on the execution of 
a response on the basis of previously experienced local, as opposed to distal, visual cues. Finally, Cho and Kesner (1996) have recently shown that extensive bilateral parietal lesions result in a temporally nongraded retrograde amnesia for preoperatively learned spatial problems. The generality of their results to the present paradigm was investigated here. After completing all performance probes, sham controls were retrained on the original discrimination, prepared with bilateral PPC lesions, and tested for retention of the habit beginning 5 days after surgery.

\section{METHOD}

\section{Subjects and Surgery}

Twenty Long-Evans strain hooded rats bred in our colony and ranging in age from 232 to 326 days at the time of surgery were used as subjects. Five animals were quasi-randomly assigned to each of the four surgical conditions. All animals were male in the sham, left-PPC, and right-PPC groups. Three of the 5 animals in the bilateral-PPC group were female. We judged this to be acceptable because, in a previous study with PPC lesions (McDaniel \& Wall, 1988), we failed to observe significant differences between males and females prepared with this lesion. The animals were individually housed in stainless steel rodent cages, and they were entrained to a reversed light:dark cycle with light offset at 8:00 a.m. and onset at 8:00 p.m. All surgical and behavioral procedures were conducted during the dark phase. Food and water were available ad lib except for $24 \mathrm{~h}$ prior to surgery. All aspects of the methodology were reviewed and approved by the Institutional Animal Care and Use Committee.

The surgical protocol has been described in greater detail previously (Davis \& McDaniel, 1993). Following intraperitoneal injections of atropine $(0.35 \mathrm{mg} / \mathrm{kg})$ and sodium pentobarbital $(45 \mathrm{mg} / \mathrm{kg})$, the scalp was shaved and scrubbed with a $10 \%$ iodine solution. The animal was mounted in a Kopf stereotaxic instrument. A midline incision was made to expose the dorsal cranium, the scalp was retracted laterally, and the dorsal-most fibers of the temporalis muscle were displaced from the temporal crest. For animals assigned to the sham control group, surgery was terminated at this point. The wound was closed with wound clips and treated with Mycitracin triple antibiotic with lidocaine. For animals receiving various PPC lesions, the dorsal cranium was drilled with a 3.5 -mm-diameter trephine centered $3.0 \mathrm{~mm}$ posterior to the coronal suture and $3 \mathrm{~mm}$ lateral to the sagittal suture. The craniotomy was enlarged with microronguers laterally to the temporal crest. The dura was incised with a dissection knife, and the underlying cortical mantle was aspirated through a glass micropipette. A $3 \times$ jeweler's loop was used to magnify the surgical field. The cavity was packed with Gelfoam as needed and the wound closed as described for sham control rats. The animals placed near a space heater until recovery from anesthesia was complete.

\section{Histology}

Upon the completion of behavioral training and assessment, the animals were anesthetized with an overdose of sodium pentobarbital $(0.5 \mathrm{ml})$ and were perfused through the heart with isotonic saline $(0.09 \%)$ followed by $50 \mathrm{cc}$ of $10 \%$ formalin. The brains were removed and stored in $10 \%$ formalin before being photographed from the dorsal perspective and transferred to a $30 \%$ sucrose in $10 \%$ formalin solution. Several days later, the tissues were blocked to include the lesion site and thalamus, and the brains were frozen and sectioned through the coronal plane in $50-\mu \mathrm{m}$ slices. Every fifth section was mounted on a slide and stained with cresyl-violet acetate. The sections were examined through a microscope for evidence of retrograde degeneration in the thalamus, for accuracy of lesion placement, and for lesion depth. The dot-grid technique was employed to estimate the relative surface area involved in creating the lesions (Thomas \& Peacock, 1965).

\section{Apparatus and Stimuli}

All training was conducted in a white circular water tank (1.4-m diameter $\times 0.6-\mathrm{m}$ height) made of galvanized steel. Galvanized steel partitions forming the shape of a $\mathrm{T}$ were fitted to the tank. The T-maze consisted of a stem, measuring $53 \mathrm{~cm}$ long $\times 30 \mathrm{~cm}$ wide, with two arms, each measuring $56 \mathrm{~cm}$ long $\times 28 \mathrm{~cm}$ wide. The apparatus was filled with water to a depth of $20 \mathrm{~cm}$. White nontoxic paint (Artistica II tempera, Hazelton, PA) was suspended in the water to obscure view of a white escape platform $(26 \times 17 \times$ $17 \mathrm{~cm}$ ) submerged $2 \mathrm{~cm}$ below the water surface. The visual stimuli consisted of identical, individual $12-\mathrm{V}$ d.c. dome lights $(7 \times$ $4.5 \mathrm{~cm}$ ) connected to a Micronta No. 22-121 adjustable dual-tracking d.c. power supply (1 A maximum output, $0-15 \mathrm{~V}$ ). A set of four lights each lined the left and right inner walls and entrances into the goal alleys. Two lights were located along the inner walls of the starting alley, and two lights were located along the inner wall of each goal alley. Each series of lights could be controlled independently by double-pole toggle switches to flash off and on at a rate of $1.2 \mathrm{cps}$, to shine constant, or to remain off. Single-pole toggle switches allowed the trainer to turn off individual lights to study performance under conditions of reduced cue saliency and increased spatial discontiguity. The lights were $15 \mathrm{~cm}$ above the water surface. The distance between the start point and the first light in each series was $24 \mathrm{~cm}$ (approximately a rat's body length), the distance between the first two lights in each series was $13 \mathrm{~cm}$, the distance between the third and fourth lights located in the goal arms was $6 \mathrm{~cm}$, and the distance between the platform and the fourth light of each series was $12 \mathrm{~cm}$. Other than the lights within the T-maze, the only light in the windowless training room was provided by a single $25-\mathrm{W}$ red light bulb and a small amount of light coming through a crack between the door and hallway to the laboratory.

\section{Behavioral Methods}

All animals were given at least 11 days of postoperative recovery prior to pretraining in the water T-maze. The animals were assigned random numbers as identifiers so that each trainer (i.e., the first three authors) would be blind to a particular animal's experimental condition. Initially, the rats were pretrained for several days to swim to escape platforms located randomly in either the right or the left goal area. This was done to reduce the likelihood that an animal might reenter the starting alley during a training trial. Thus, exposure to the visual cues was restricted to only one visual field within a trial.

Each trial consisted of placing the rat into the maze with its body close to the end wall of the starting alley and its head facing into the maze. A Fellows (1967) series was used to randomize the spatial location of the escape platform across trials. The animals were allowed to search both arms of the maze without penalty until escape was achieved. Errors were defined as an entry into the incorrect alley to within $16 \mathrm{~cm}$ of where the front edge of the escape platform would have been located on a correct trial. During training, flashing lights along the right wall of the starting alley and along the entrance to the right goal alley cued that the escape platform was in the right alley. Likewise, flashing lights on the left walls cued the location of the platform in the left alley.

All animals were trained until they achieved at least $85 \%$ correct choices in a single daily session of 20 trials. On the following day, performance was assessed with the light cue closest to the escape platform turned off. Thus, location of the escape platform had to be determined by the signals provided in the starting alley and by the single light in the goal alley located closest to the choice area. On the next day, performance was assessed with both lights in the goal alleys turned off. This condition required the rats to execute a spatial response on the basis of information contained along the inner wall 
of the starting alley. Finally, 3 additional days of testing were conducted with competing stimuli. That is, the flashing of four lights along the right or left inner walls of the start and goal alleys cued position of the escape platform. The lights along the opposite wall were constantly on but not flashing. Following a 2-week break, 3 of the sham control rats were retrained to criterion on the original visuospatial task, prepared with bilateral PPC lesions, and later tested for retention of the turn-signal response habit.

\section{RESULTS}

\section{Lesion Analysis}

Photographs depicting representative lesions from the dorsal perspective are shown in Figure 1. The locations of the lesions appear to be consistent with those reported in previous studies (e.g., King \& Corwin, 1992; Kolb \& Walkey, 1987; McDaniel et al., 1995; McDaniel \& Wall, 1988). The size of each lesion was estimated using the dot-grid method (Thomas \& Peacock, 1965). The MannWhitney $U$ test confirmed that the unilateral left and unilateral right lesions were similar in size $(U=5.00, z=$ $1.57, p=.11$ ).

Examination of the cresyl-violet stained coronal sections through the lesions showed that the depth and pattern of thalamic degeneration were nearly identical to those depicted in our previous studies (e.g., McDaniel et al., 1995). Figure 2 shows reconstructions of the le-
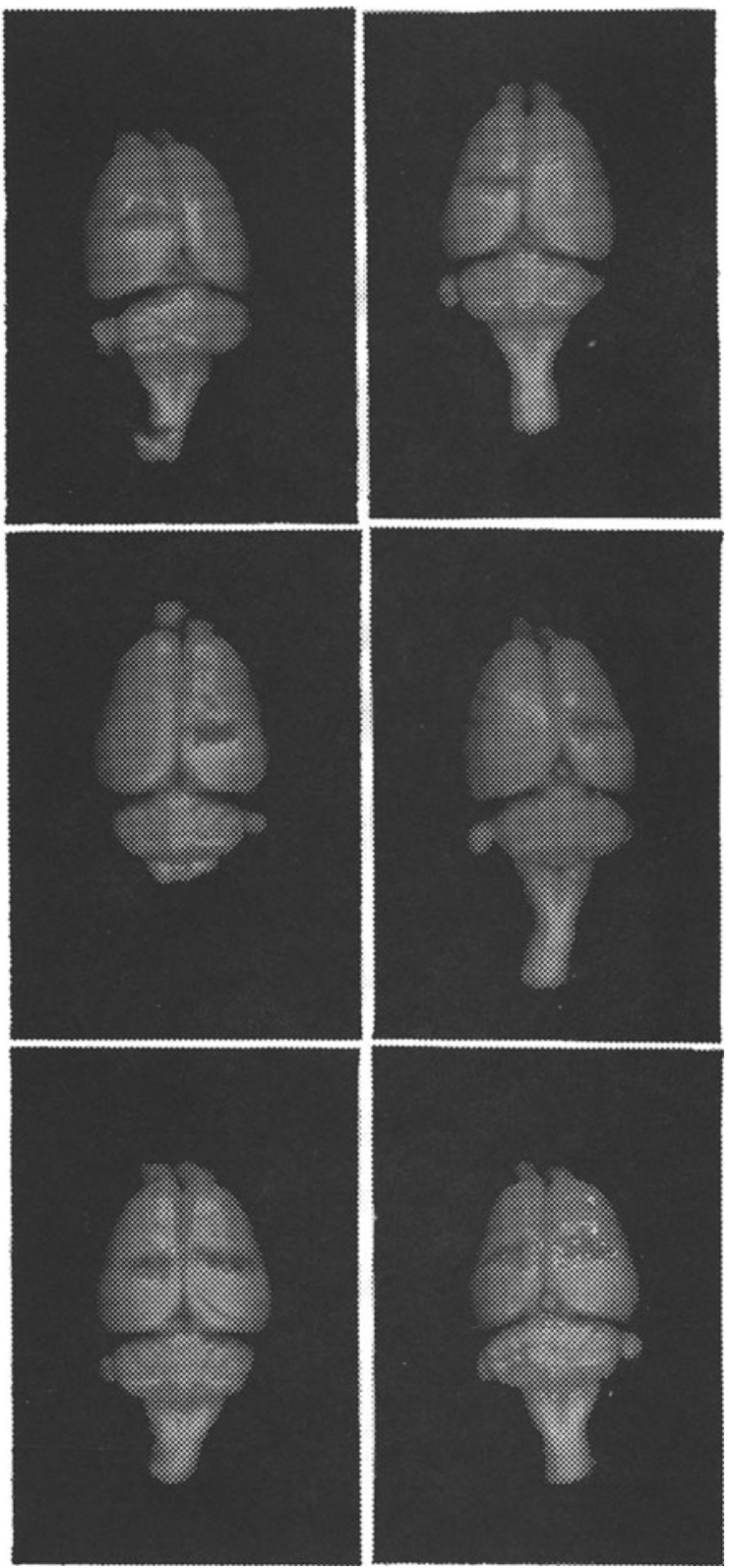

Figure 1. Photograph of the lesions in representative cases, viewed from the dorsal perspective. 


\section{Unilateral Lesions}

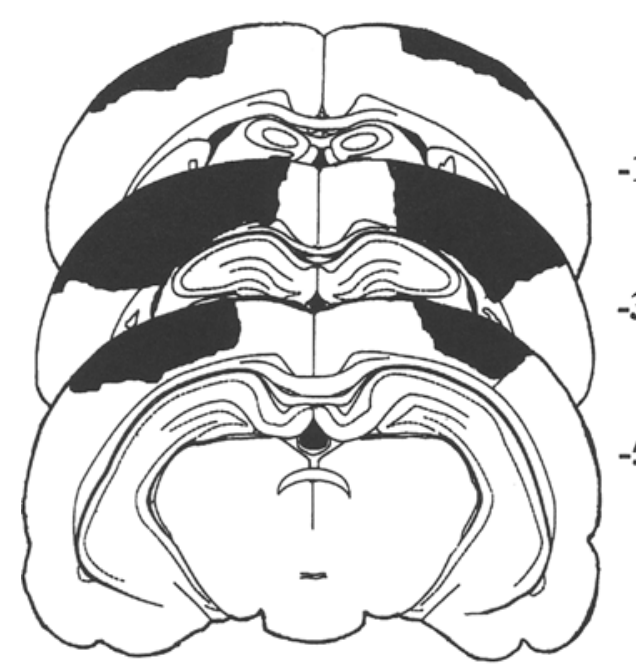

Bilateral Lesions

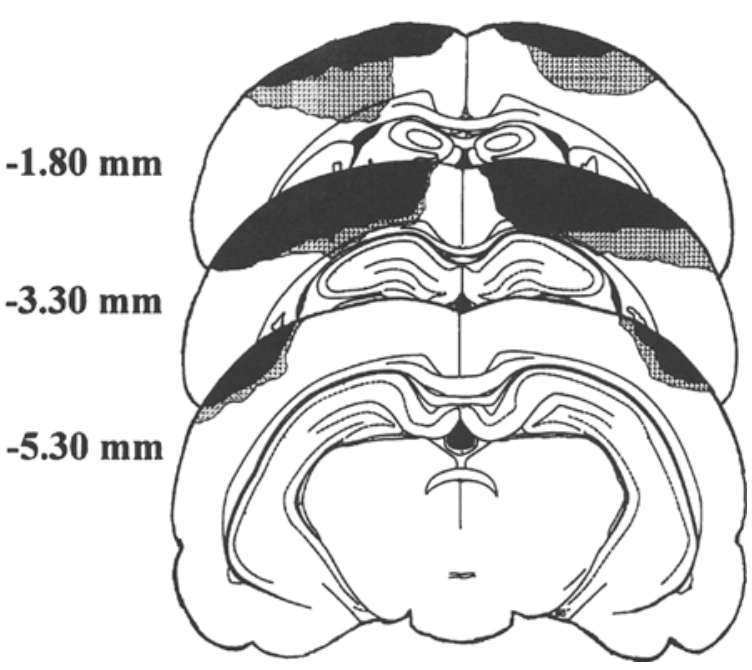

Figure 2. Reconstructions of the lesions as revealed from an analysis of the histological material.

sions as revealed through an examination of the histological material. In almost all cases, the lesions produced damage to underlying white matter. However, in no case was the hippocampal formation directly damaged. Moderate gliosis was apparent in the lateral and posterior thalamic nuclei. Occasionally, pockets of focal dense gliosis were observed in the ventral complex. This could have been due to the damaged white matter or the slight invasion of the hindlimb or PAR 1 region observed in some cases. In no case was there evidence of gliosis in the lateral geniculate nuclei.

\section{Behavior Analyses}

A one-way analysis of variance (ANOVA) confirmed that there were no overall differences in the number of pretraining (working memory) errors across the four lesion groups $[F(3,17)=1.31, p=.30]$. Also, one-way ANOVAs were used to examine the total number of errors and trials through criterion on the flashing-light-spatialchoice discrimination. Both ANOVAs resulted in significant main effects $[F \mathrm{~s}(3,16)=8.34$ and $4.24, p s=.0014$ and .0219 , respectively]. With respect to spatial-choice errors, the Fisher's LSD multiple-comparisons test showed that the rats with bilateral injuries committed significantly more errors while learning the discrimination than did all other groups. Also, the animals with left-hemisphere injuries committed significantly more errors than did the sham control group. The Fisher's LSD multiple-comparisons test showed that the rats with bilateral injuries required more trials to reach criterion than did the other groups (see Figure 3).

Two-way split-plot ANOVAs (SPANOVAs) were used to evaluate the number of errors committed by the lesion groups as a function of the position of the left/right locations of the flashing-light stimuli and left/right spatial choices required. As expected from the foregoing analyses, this analysis resulted in a significant main effect for lesion $[F(3,16)=8.49, p=.0013]$. However, there were no overall differences across groups in the number of errors made when the stimuli were located on the right versus the left side $[F(1,16)=0.14, p \gg .05]$, and there was no interaction between the lesion and errors made when the stimuli and required responses were left or right $[F(3,16)=0.77, p \gg .05]$. The latter result demonstrates that, regardless of the lesion condition, in the course of training, the animals with lateralized lesions utilized stimuli located in the left and right visual fields as well as the sham control animals did. A SPANOVA was also used to analyze errors committed when stimuli were in the left or the right visual field for the final 60 trials through the criterion swim. This analysis revealed only one significant effect. This was a main effect for location of the flashinglight stimuli and response required (i.e., a main effect for the repeated measures variable) $[F(1,16)=5.56, p=$ $.0314]$. This result revealed that, across all groups, the rats tended to make more errors when required to select the left alley than when required to select the right alley. We are uncertain why this occurred, but we initially hypothesized that the location of the apparatus in the room with respect to the low level of illumination available may have produced this transient right-alley bias just prior to attaining criterion. However, LaMendola and Bever (1997) have reported a slight but significant right-turn bias in rats. The authors suggest tentatively that this result may reflect a left-hemisphere and right-sensory experiential bias in utilizing sensory/perceptual stimuli in the negotiation of novel spatial environments. The main effect for lesion and the interaction of lesion $\times$ location of the stimulus/ response were nonsignificant (both $p$ s $\gg .05$ ). Combined with the results of the previous analysis, the lack 

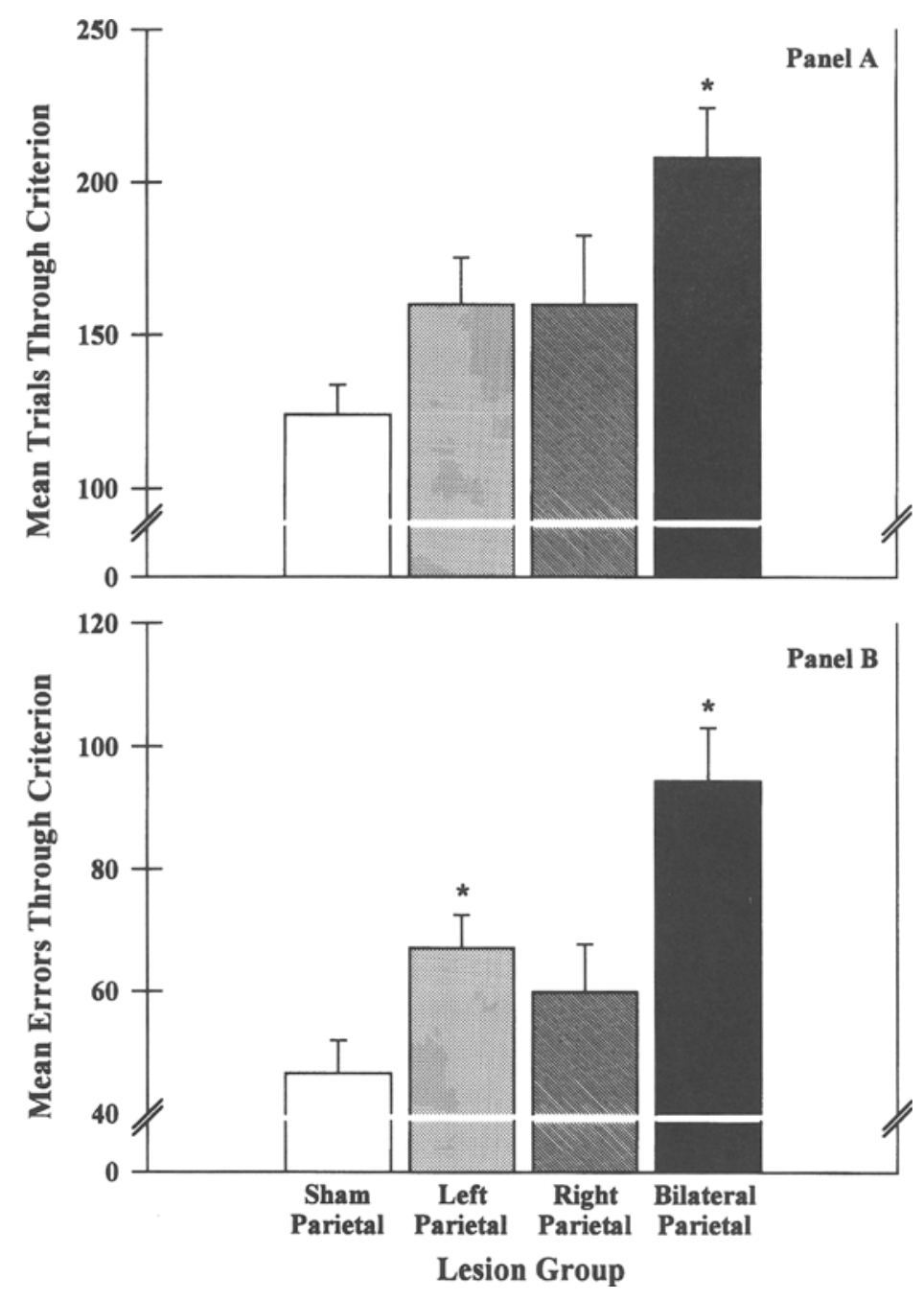

Figure 3. Panel A: Mean and standard error of the mean trials through criterion for the lesion groups while learning the flashing-light-spatial-turn habit. Panel B: Mean and standard error errors made by the lesion groups while learning the flashing-light-spatial-turn habit. Asterisks indicate a significant elevation with respect to the sham control group.

of an interaction in both studies indicates that the animals with unilateral injuries had no greater difficulty using stimuli contralateral to the lesion than they did using stimuli contralateral to the intact hemisphere to guide spatial behavior. This result is consistent with King and Corwin (1992), who reported that animals with unilateral PPC lesions failed to show hemispatial visual neglect in their egocentric and allocentric spatial tasks.

A three-way SPANOVA with two repeated measures (lesion location $\times$ task $\times$ left vs. right alley entrances) was used to examine the number of errors committed when performance of the spatial task was assessed under conditions of reduced stimulus saliency. Specifically, on the day after the session in which criterion was attained, the flashing light closest to the escape platform was turned off. Therefore, two lights in the starting alley and one light at the entrance to the correct goal alley signaled location of the escape platform. On the next day, both goal-alley lights were turned off. This required the rat to make a spatial response on the basis of the two flashing lights in the starting alley. The main effect for lesion was nonsignificant $(p \gg .05)$. However, the main effect for task was significant $[F(1,16)=87.01, p<.0001]$. This result was expected. It showed that executing the spatial response solely on the basis of visual cues located in the start alley was much more difficult than executing the spatial response on the basis of these stimuli plus one additional light stimulus available at the entrance to the correct goal alley. Also, the interaction of lesion group $\times$ task was significant $[F(3,16)=3.15, p=.05]$. This result, shown graphically in Figure 4, was due to the greater number of errors made by the animals with left PPC lesions and bilateral PPC lesions, relative to the animals with sham or right PPC injuries, when required to utilize information 


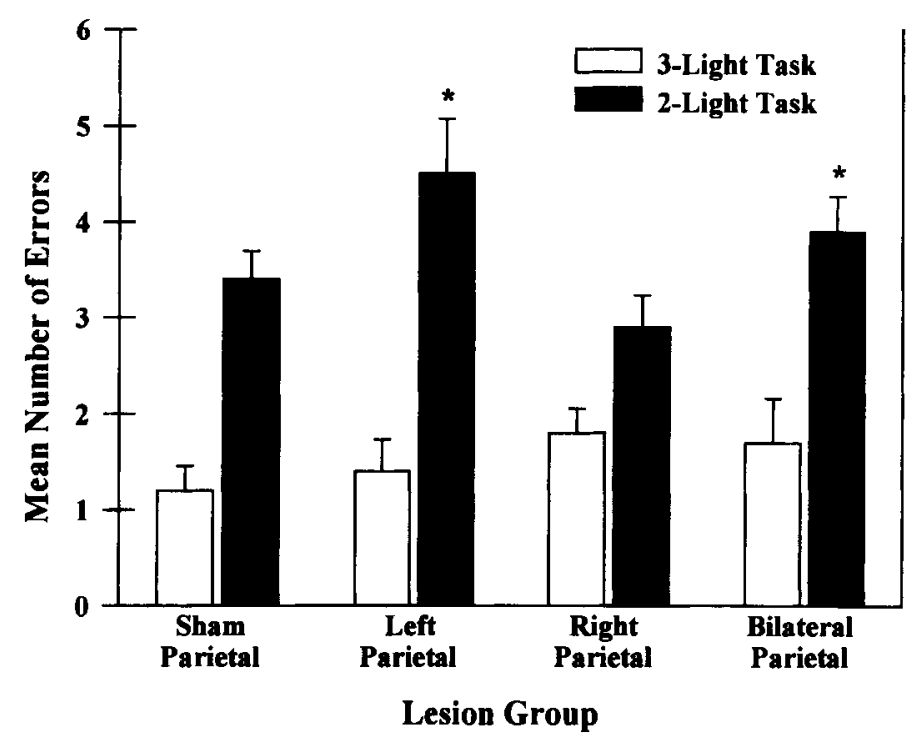

Figure 4. The means and standard errors of the mean performance errors under conditions of decreased cue saliency and increased spatial discontiguity. In the two-light task, the spatial choice made in the choice area was based on cues provided in the starting alley. Asterisks indicate significant differences relative to the sham control and right PPC injured groups.

in the starting alley to make the correct spatial response. Of course, identical statistical results were observed in analyses of choice accuracy (i.e., proportion correct responses).

A two-way SPANOVA (lesion $\times$ trial block) was also used to assess the number of errors committed across the three sessions of performance testing with competing stimuli. In this case, all four flashing lights along the inner walls cued the location of the escape platform, as had been the case in the initial task. However, the four lights on the opposing walls were constantly on. In this condition, the performance of almost all animals plummeted to chance levels and failed to improve across sessions. Neither the main effects nor the interaction were significant.

Finally, 3 of the sham control rats were retrained on the original flashing-light-spatial-choice discrimination and then were prepared with bilateral PPC lesions. With respect to savings scores calculated on errors, considerable postoperative retention was observed in all three cases $(59 \%, 74 \%$, and $86 \%)$. Using the $t$ test for correlated samples, the number of retraining errors prior to surgery was compared with the number of retraining errors 5 days after surgery. This analysis showed that the animals made fewer postoperative errors than they did in the presurgical retraining sessions $[t(2)=6.28, p<.01]$.

\section{DISCUSSION}

The aim of the present study was to investigate the influences of unilateral or bilateral PPC injuries upon the acquisition and performance of a visually cued spatial habit. In initial training, the rats were required to select the right or the left goal alley in a water T-maze on the basis of local visual cues. That is, flashing turn-signal lights, presented along the inner walls of the starting alley and correct goal alley, cued the location of the submerged escape platform. Once learned, the saliency of the cues was diminished while the spatial discontiguity between the stimuli and escape platform was increased in two steps (i.e., the three-light and two-light tasks).

The rats with bilateral PPC injuries committed more errors and required more trials to achieve criterion on the initial habit than did all other groups. Once achieving criterion, they continued to perform well when the light along the inner goal wall closest to the escape platform was turned off. However, the performance of this group was substantially impaired when both lights in the goal alley were turned off, thus requiring the rats to utilize visual cues in the starting alley in order to execute the correct right or left turn at the choice point.

The rats with left-hemisphere injuries performed much like those with bilateral injuries. Although they did not require significantly more trials than the animals with right PPC or sham injuries, the left-PPC-injured rats made significantly more errors than did the sham control animals while learning the original discrimination. Also, like the other groups, their performance of the habit was not seriously impaired when the light closest to the escape platform no longer signaled the correct alley. However, like the animals with bilateral PPC injuries, they made significantly more errors than did the sham control and right-PPC-injured rats when required to select a goal alley on the basis of visual information in the starting 
alley. Curiously, the animals with right PPC injuries were not significantly variant from the sham control animals on any of the tasks.

Previous research (Crowne et al., 1992; King \& Corwin, 1992) has shown a special role of the right PPC in spatial navigation on the basis of allocentric distal cues. This result is consistent with observations made in humans who have sustained right posterior association cortex injuries (e.g., Kolb \& Whishaw, 1996; Weintraub \& Mesulam, 1987). We have argued likewise on the basis of our investigations using the Greek-cross water-maze task (McDaniel et al., 1995). That is, we found that rats with unilateral or bilateral PPC lesions acquired a placelearning habit as rapidly as controls did; however, animals with bilateral PPC and selective right PPC injuries were significantly inferior to the animals with left PPC injuries and the sham controls. We suggested that the response-learning task might have been mastered either by learning to make a specific response at the choice point or by learning two nonconceptual conditional associations between stimuli (e.g., if approaching extramaze stimuli visible from starting alley one, turn toward the extramaze stimuli associated with goal alley one, etc.). Perhaps injuries in both hemispheres disturb the egocentric processes responsible for response learning. However, the animals with an intact right hemisphere may be able to compensate for the egocentric dysfunction by using extramaze visual cues. A second experiment was conducted to test this hypothesis. Rats with unilateral or sham PPC lesions were trained on the response-learning task in the absence of reliable visual cues. Under these conditions, the rats with left PPC injuries performed similar to those with right PPC injuries, and both groups were significantly impaired in learning relative to sham control animals. These conclusions appear to be consistent with those reached by Save and Moghaddam (1996), who found that rats with bilateral lesions of the APC show "egocentric" deficits in a spatial navigation task trained in total darkness. Collectively, these results support the argument that the PPC in both hemispheres plays a role in the integration of kinesthetic information necessary for egocentric spatial processes.

The results of the present experiment suggest that the left PPC plays a greater role than the right PPC in the use of intramaze or local visual cues (as in the turn signals used here) for spatial navigation. This result appears to be consistent with the findings of LaMendola and Bever (1997), who reported, using both unilateral spreading cortical depression and unilateral whisker anesthesia, that the left hemisphere is more involved in the coding of local cues while learning a novel foraging task. Additionally, they reported that the right hemisphere played a greater role in coding the more global cues associated with a novel environment. As was discussed previously, lesions of the right PPC have been shown to disturb spatial navigation on the basis of distal visual cues in several studies
(Crowne et al., 1992; King \& Corwin, 1992; McDaniel et al., 1995). Thus, collectively, it appears that rats with an intact right PPC, but an injured left PPC, can utilize distal visual cues to guide spatial navigation. However, animals with this injury are impaired in their use of local visual cues. On the other hand, rats with an intact left PPC, but an injured right PPC, are better able to utilize local visual cues for purposes of spatial navigation. However, animals with this injury are deficient in the use of distal environmental cues.

The deficit observed in the turn-signal cued-response task by the bilateral- and left-PPC-injured rats cannot be explained as a generalized failure of working memory. This is because analyses of pretraining performance failed to show differences in alley reentries for any group. Also, in a previous study (McDaniel et al., 1994), workingmemory errors committed in a multiple-T water maze were examined for rats prepared with either bilateral PPC, hippocampal, or sham injuries. Only the hippocampectomized rats made significantly more workingmemory errors.

Finally, Cho and Kesner (1996) have shown that extensive bilateral parietal lesions result in a temporally nongraded "retrograde amnesia" for spatial habits learned in the eight-arm radial maze. The substantial savings observed in our sham animals later prepared with bilateral PPC lesions indicate that the PPC is, at best, only a small part of the neural system involved in long-term storage of such relationships.

\section{REFERENCES}

Boyd, M. G., \& Thomas, R. K. (1977). Posterior association cortex lesions in rats: Mazes, pattern discrimination, and reversal learning. Physiological Psychology, 5, 455-461.

Burcham, K. J., Corwin, J. V., Stoll, M. L., \& Reep, R. L. (1997). Disconnection of medial agranular and posterior parietal cortex produces multimodal neglect in rats. Behavioural Brain Research, 86, 41-47.

Chandler, H. C., King, V., Corwin, J. V., \& Reep, R. L. (1992). Thalamocortical connections of rat posterior parietal cortex. Neuroscience Letters, 143, 237-242.

Chen, L. L., Lin, L.-H., Barnes, C. A., \& McNaughton, B. L. (1994). Head-direction cells in the rat posterior cortex: II. Contributions of visual and ideothetic information to the directional firing. Experimental Brain Research, 101, 24-34.

Chen, L. L., Lin, L.-H., Green, E. J., Barnes, C. A., \& McNaughton, B. L. (1994). Head-direction cells in the rat posterior cortex: I. Anatomical distribution and behavioral modulation. Experimental Brain Research, 101, 8-23.

Cho, Y. H., \& KeSNER, R. P. (1996). Involvement of entorhinal cortex or parietal cortex in long-term spatial discrimination memory in rats: Retrograde amnesia. Behavioral Neuroscience, 110, 436-442.

Crowne, D. P., Novotny, M. F., Maier, S. E., \& Vitols, R. (1992). Effects of unilateral parietal lesions on spatial localization in the rat. Behavioral Neuroscience, 106, 808-819.

Crowne, D. P., Richardson, C. M., \& Dawson, K. A. (1987). Lateralization of emotionality in right parietal cortex of the rat. Behavioral Neuroscience, 101, 134-138.

DAVIS, B. K., \& MCDANIEL, W. F. (1993). Visual memory and visual spatial functions in the rat following parietal and temporal cortex injuries. Physiology \& Behavior, 53, 145-151. 
DiMatTIA, B. V., \& KeSNER, R. P. (1988a). Role of the posterior parietal association cortex in the processing of spatial event information. Behavioral Neuroscience, 102, 397-403.

DiMatTia, B. V., \& KeSNER, R. P. (1988b). Spatial cognitive maps: Differential role of parietal cortex and hippocampal formation. Behavioral Neuroscience, 102, 471-480.

Fellows, B. J. (1967). Chance stimulus sequences for discrimination tasks. Psychological Bulletin, 67, 87-92.

Hyvärinen, J., \& Poranen, A. (1974). Function of the parietal associative area 7 as revealed from cellular discharges in alert monkeys. Brain, 97, 673-692.

Jucker, M., Kametani, H., Bresnahan, E. L., \& Ingram, D. K. (1990). Parietal cortex lesions do not impair retention performance of rats in a 14 unit $\mathrm{T}$ maze unless hippocampal damage is present. Physiology \& Behavior, 47, 207-212.

Kesner, R. P., Farnsworth, G., \& DiMattia, B. V. (1989). Double dissociation of egocentric and allocentric space following medial prefrontal and parietal cortex lesions in the rat. Behavioral Neuroscience, 103, 956-961.

KING, V. R., \& CoRwin, J. V. (1992). Spatial deficits and hemispheric asymmetries in the rat following unilateral and bilateral lesions of posterior parietal or medial agranular cortex. Behavioural Brain Research, 50, 53-68.

KoLB, B. (1990). Posterior parietal and temporal association cortex. In B. Kolb \& R. C. Tees (Eds.), The cerebral cortex of the rat (pp. 459471). Cambridge, MA: MIT Press.

Kolb, B., Buhrmann, K., McDonald, R., \& Sutherland, R. J. (1994). Dissociation of the medial prefrontal, posterior parietal, and posterior temporal cortex for spatial navigation and recognition memory in the rat. Cerebral Cortex, 6, 664-680.

Kolb, B., Sutherland, R. J., \& WhishaW, I. Q. (1983). A comparison of the contributions of the frontal and parietal association cortex to spatial localization in rats. Behavioral Neuroscience, 97, 13-27.

KOLB, B., \& WALKEY, J. (1987). Behavioural and anatomical studies of the posterior parietal cortex in the rat. Behavioural Brain Research, 23, 127-145.

KoLB, B., \& WhishaW, I. Q. (1996). Fundamentals of neuropsychology (4th ed.). New York: Freeman.

LaMendola, N. P., \& Bever, T. G. (1997). Peripheral and cerebral asymmetries in the rat. Science, 278, 483-486.

LASHLEY, K. S. (1941). Thalamo-cortical connections of the rat's brain. Journal of Comparative Neurology, 75, 67-121.

MCDANIEL, W. F. (1985). Functions of the posterior neocortex of the rat. IRCS Medical Science, 13, 286-289.

McDaniel, W. F., Compton, D. M., \& SMith, S. R. (1994). Spatial learning following posterior parietal or hippocampal lesions. NeuroReport, 5, 1713-1717.

MCDaniel, W. F., Davall, E. J., \& Waters, P. E. (1989). ACTH 4-9 analog can retard spatial alternation learning in brain damaged and normal rats. Behavioral \& Neural Biology, 52, 271-278.

McDaniel, W. F., McDaniel, S. E., \& Thomas, R. K. (1978). Thalamocortical projections to the temporal and parietal association cortices in the rat. Neuroscience Letters, 7, 121-125.

MCDANIEL, W. F., \& SKeEL, R. L. (1993). Visual-spatial functions persist following temporal and posterior parietal cortex lesions in rat. Physiology \& Behavior, 53, 199-203.
MCDaniel, W. F., \& Thomas, R. K. (1978). Temporal and parietal association cortex lesions and spatial and black-white reversal learning in the rat. Physiological Psychology, 6, 300-305.

McDaniel, W. F., Via, J. D., Smith, J. S., Wells, D. L., Fu, J. J., Bishop, J. F., BoYd, P. A., \& Ledesma, H. M. (1995). Unilateral injury of posterior parietal cortex and spatial learning in hooded rats. Behavioural Brain Research, 70, 165-179.

MCDANIEL, W. F., \& WALL, T. T. (1988). Visuospatial functions in the rat following injuries to striate, peristriate, and parietal neocortical sites. Psychobiology, 16, 251-260.

McNaughton, B. L., Leonard, B., \& Chen L. [L.] (1989). Corticalhippocampal interactions and cognitive mapping: $A$ hypothesis based on reintegration of the parietal and inferotemporal pathways for visual processing. Psychobiology, 17, 236-246.

Reep, R. L., Chandler, H. C., King, V., \& Corwin, J. V. (1994). Rat posterior parietal cortex: Topography of corticocortical and thalamic connections. Experimental Brain Research, 100, 67-84.

Save, E., Buhot, M.-C., Foreman, N., \& Thinus-Blanc, C. (1992). Exploratory activity and response to a spatial change in rats with hippocampal or posterior parietal cortical lesions. Behavioural Brain Research, 47, 113-127.

Save, E., \& Moghaddam, M. (1996). Effects of lesions of the associative parietal cortex on the acquisition and use of spatial memory in egocentric and allocentric navigation tasks in the rat. Behavioral Neuroscience, 110, 74-85.

Save, E., Poucet, B., Foreman, N., \& Buhot, M.-C. (1992). Object exploration and reactions to spatial and nonspatial changes in hooded rats following damage to parietal cortex or hippocampal formation. Behavioral Neuroscience, 106, 447-456.

Thinus-Blanc, C., Save, E., Poucet, B., \& Foreman, N. (1996). Effects of parietal cortex lesions on spatial problem solving in the rat. Behavioural Brain Research, 81, 115-121.

Thomas, R. K., \& Peacock, L. J. (1965). A method for measuring brain lesions. Psychological Science, 3, 184.

Thomas, R. K., \& WeiR, V. K. (1975). The effects of lesions in the frontal or posterior association cortex of rats on Maze III. Physiological Psychology, 3, 210-214.

Thompson, R., Heustis, P. W., Crinella, F. M., \& Yu, J. (1986). The neuroanatomy of mental retardation in the white rat. Neuroscience \& Biobehavioral Reviews, 15, 317-338.

ThOMPSON, R., \& Yu, J. (1985). The comparative effects of frontal, parietal, occipitotemporal, and limbic forebrain lesions in weanling rats on learning. Physiology \& Behavior, 15, 559-567.

VogT, B. A., \& MilLER, M. W. (1983). Cortical connections between rat cingulate cortex and visual, motor, and postsubicular cortices. Journal of Comparative Neurology, 216, 192-210.

Weintraub, S., \& Mesulam, M.-M. (1987). Right cerebral dominance in spatial attention: Further evidence for ipsilateral neglect. Archives of Neurology, 44, 621-625.

ZiLLES, K. (1985). The cortex of the rat: A stereotaxic atlas. Berlin: Springer-Verlag.

(Manuscript received September 15, 1997; revision accepted for publication December 30, 1997.) 